\title{
A Study of Car Sharing in China Based on Evaluation Index System
}

\author{
Zan $\mathrm{Li}^{1,2}$, Fuquan Zhao ${ }^{1,2}$, Zongwei Liu ${ }^{1,2,3, a}$ \\ ${ }^{1}$ State Key Laboratory of Automotive Safety and Energy, Tsinghua University, Beijing 100084, China \\ ${ }^{2}$ Tsinghua Automotive Strategy Research Institute, Tsinghua University, Beijing 100084, China \\ ${ }^{3}$ Sloan Automotive Laboratory, Massachusetts Institute of Technology, Cambridge MA 02139, USA
}

\begin{abstract}
The popularization of automobiles is originally a symbol of social progress, but it has also brought many problems and hidden dangers to the urban development of China in the aspects of transportation, environment, and energy. In this context, the emergence of the car sharing has led people from all walks of life to discover ways to solve these problems. This paper first studies and reveals the significance of car sharing. Then, on the basis of the development bottleneck of car sharing, this paper builds an evaluation index system to objectively analyze and evaluate the development status and find out the keys to solving the problems rooted in the car sharing status in China. Finally, this paper puts forward specific strategies and suggestions to promote the formation of China's car-sharing society.
\end{abstract}

\section{Introduction}

China's urbanization rate has been growing continuously, which is expected to exceed $70 \%$ by 2035 [1]. The rapid development of urbanization is constantly changing the social landscape, which is reflected in the significant increase in traffic trips and car ownership. The nature of transportation is to meet people's demands for mobility [2]. Due to urban expansion, population aggregation and other factors, the demand for urban transportation will continue to grow in the future, which will lead to diversified mobility modes and the emergence and blooming development of new mobility modes and new business forms to share the original traffic flow, among which is car sharing. The increase in car ownership resulted in the consumption of urban space, such as road space and parking spaces, which posed a serious challenge to the resource supply capacity and resource allocation capacity of cities [3]. Car sharing can be an effective way to deal with the problem of strained road resources, and can also be a key trick to build a smart and clean urban transportation network.

Car sharing fosters the public's habit of using cars on demand by centrally providing shared vehicles, thereby reducing vehicle ownership. Munich launched more than 550 vehicles for car-sharing services, which saved more than 1,500 social parking spaces for the city, increased the proportion of bus trip by $14 \%$ and reduced private car trips by about $25 \%$ [4]. One Car2go's vehicle can curb the purchase demand for 4-9 private cars, according to the data released by the company [5]. According to the study by Michiko and other scholars in Vancouver, the average vehicle ownership rate of families dropped from 1.08 to 0.98 units after Car2go platform was locally

\footnotetext{
a Corresponding author: liuzongwei@tsinghua.edu.cn
}

available for those using car sharing service. The rate dropped from 0.68 to 0.36 units after the arrival of Modo, another car sharing platform [6]. According to the study by Scott and other academics in London, $37 \%$ of the users indicated that free-floating car sharing affected their ownership of private cars after joining local free-floating car sharing platform [7]. Since the gap rate of average parking spaces in first- and second-tier cities in China is now generally high [8], whether reducing parking time of purchased vehicles, increasing the share rate of public transportation, reducing private car trips, or discouraging the purchase demand for private cars can have positive impact on easing the strain on road and parking resources.

Car sharing enhances the driving and riding efficiency. The congestion-relieving effects of car sharing are supported by numerous studies. According to a study, shared mobility can solve nearly $10 \%$ of urban congestion problems. The study also cited MIT's research that carpooling can reduce traffic congestion by $55 \%$ and that a fully functioning "shared car" can replace approximately 4-10 private cars [9]. Another study showed that every vehicle for car sharing can replace 20 ordinary cars [4]. According to data released by Car2go, one of the company's cars can replace $7-11$ private cars and reduce travelled distance by $6-16 \%$ [5]. The improvement of traffic conditions brought by car-sharing is the consequence both of curbing the absolute growth of car ownership through the reduction of private car purchases and rational increase of the turnover efficiency of traffic flows. Both mechanisms work together to enhance vehicle use and improve traffic flow.

Car sharing can weaken the impact on the environment. First, merging trips increases travel efficiency and indirectly reduces energy consumption and 
pollution emissions from redundant trips. One Car2go's car can reduce greenhouse gas emissions by $4-18 \%$ [5]. Second, keeping the driving speed within a reasonable range can also facilitate sufficient burning of gasoline and reduce the pollution emissions from insufficient oxidation. According to the study by Jörg, et al. of Car2go users in Ulm, Germany, 146 kg-312 kg CO 2 emissions can be reduced by every user annually under different scenarios [10]. According to Martin's calculation of the impact on the household level, $580 \mathrm{~kg} \mathrm{CO}_{2}$ emissions can be reduced by every family annually, which, if divided by the average household size of 2.5 in the region, equates to a reduction of $232 \mathrm{~kg} \mathrm{CO}$ emissions per person per year [11]. In addition, the car-sharing industry, which uses a large number of new energy vehicles (NEV), has fundamentally achieved low-carbon travel from vehicle power. Guided by the two goals of building a strong automotive industry and low-carbon development in China, the development of low-carbon automotive industry is an inevitable choice for national and industrial development [12]. Considering the low impact on environment, wide range of energy sources, high conversion rate and low tariff, electric vehicle can be an important option for urban haze management, energy conservation and carbon reduction.

Car sharing can optimize travel experience. With public transport as the guiding principle, car sharing is a complement to the lifestyle [13], which will change people's travel habits and attitudes. In the past, the overall capacity of taxis was difficult to guarantee the demand for service during peak hours, while taxi drivers would occasionally refuse passengers because of personal gain, which has become a long-standing problem in big cities. By matching both the supply and demand in a timely manner, car sharing ensures that every user can gain the access to a car wherever and whenever he or she is, which can greatly lower the risk of not having a car to ride during peak hours. In the meantime, platform-based operation can strengthen the duly management and control of drivers, which not only allows the use of big data for vehicle scheduling, but also ensures timely feedback of passengers' complaints and rights protection during driving. Car-sharing-related platform technologies ensure the timeliness and safety of car hailing for the public. As the industrial restructuring is just starting today, both new energy and intelligent connectivity technologies will bring consumers all new product experience, and will gradually build a brand-new brand identity in their minds [14], for which car sharing is becoming a concrete carrier.

However, car sharing in China is now facing a period of development bottleneck, which is mainly manifested in the slowdown of vehicle scale expansion, shutdown of business operation platforms, consumers' rejection and so on. The industry is facing an array of uncertainties for development [15]. In order to faster move into an " $24 / 7$ car-sharing" society [16] and to make the concept of "car sharing" play a valuable role in China's transportation and mobility, this paper will build a car sharing development evaluation index system to identify shortcomings and problems and explore measures for improvement based on a comprehensive evaluation of all aspects of car-sharing development in China, and provide reliable suggestions for government management decisions and corporate strategy development.

\section{Methodology}

\subsection{Research subjects and data sources}

Car-sharing development varies by regions due to economic level, manufacturing development level and infrastructure. This paper selects cities for car-sharing development from such dimensions as representativeness, comparability, and coverage. Representativeness refers to the selection of cities with relatively well-developed carsharing and local operators that are eclectic in type and have successfully passed the test of the market. Comparability means that the level of urban development, operation and R\&D capabilities of operators can be compared. Coverage is defined as the total business size of the selected operators should cover more than 80 percent of the market presence to ensure that the results can reflect the national level.

Considering all the above requirements, Beijing, Shanghai, Hangzhou, Shenzhen, Guangzhou, Chongqing, and Wuhu are selected for study in this paper, as well as typical car sharing service operators in every city, namely "MOREFUN" in Beijing, "EVCARD" in Shanghai, "Microbus" in Hangzhou, "PonyCar" in Shenzhen, "GoFun" in Guangzhou, "Panda Auto" in Chongqing, and "Eakay" in Wuhu.

After determining the study subject, this paper collects the required data via research and induction. To be specific, the data scope includes policy support, status quo of industrial technology, transportation structure, enterprise operating costs, promotion coverage, user characteristics, market landscape and other aspects. The specific approach is to collect the required information and data from academic papers on car-sharing, policy planning documents, annual reports on traffic management, reports on big data of car sharing, and other channels. The data timeframe is mainly from 2017 to 2019. Based on the refinement of data and information, this paper will conduct classification, analysis, evaluation, comparison, and trend judgment together with the carsharing development evaluation index system set forth below.

\subsection{Car-sharing development evaluation index system}

As mentioned above, this paper builds an index system to quantitatively evaluate the maturity of today's car-sharing development. Use existing problems facing the carsharing industry as a guide to identify the key factors that affect the development, which can be refined and expanded into an index system to evaluate the development degree of car sharing. Using the index system for comprehensive evaluation of the car-sharing industry can serve as a guidance for the future development of the industry. 
To be specific, this paper will draw on the ideas of the Analytic Hierarchy Process (AHP) to reflect the development of the car-sharing industry in a comprehensive, concrete, and deep manner. The AHP, proposed by American operations researcher Professor T.L. Saaty in the 1970 s, can be used to solve complex uncertainty problems, especially with multiple factors involved. The method is applied for urban planning, economic management, science and technology development evaluation and other fields. Building the index system in this paper has absorbed the core requirements of AHP for hierarchical classification of indexes, with attention paid to the hierarchical and affiliation relationship in the building of indexes at all levels. The principle in selecting the indexes is trying to be scientific, representative, forward-looking, systematic, and feasible. These indexes should meet the purpose of the study and the criteria of scientific research, with each having a specific meaning. Meanwhile, there should be a logical correlation between them to form an organic whole. Finally, there should be specific and authoritative data for support, which can be integrated through some kind of impartial calculation.

Based on the above principles and existing research base, this paper has built a car-sharing development evaluation index system. The primary indexes include five items: policy support, cost competitiveness, technological advancement, product and service experience, and business model effectiveness. The secondary indexes include 14 items, namely positioning accuracy, development support, vehicle purchase cost, business operation cost, technology upgrade cost, vehicle operating profit, intelligent connectivity technology, new energy technology, operating platform technology, convenience, safety, functionality, collaboration, and value increment. The secondary indexes are further broken down into a total of 39 specific evaluation points based on the richness of meaning.

The policy dimension is concerned with whether car sharing is given a reasonable formal status and the specific support intensity. The cost dimension can be broken down according to the business development. The technology dimension considers technologies that can better support "sharing", like autonomous driving and networking technologies, and new energy technologies. The product and service dimension addresses the user experience in terms of convenience, safety, and functionality. The business model dimension is an evaluation of collaboration between all players in the carsharing ecosphere.

\subsection{Application of the evaluation index system}

The specific evaluation points of the secondary indexes can be divided into qualitative and quantitative ones, both of which should be assigned and weighted to obtain the final primary indexes.

In the first step of assignment, qualitative index evaluation points, also called hierarchical evaluation points, can be qualitatively graded according to their specific content, with each grade corresponding to a different score. For the purposes of this paper, such indexes will be scored qualitatively within the range of $[1,5]$ based on the actual performance of each city under the index. Quantitative index evaluation points, also known as data calculation points, can be divided into two subcategories according to objective computing needs. One subcategory is the general numerical evaluation point, with which value is directly investigated and processed in a standardized manner for de-measurement and rounding. Another subcategory is the index evaluation point for the cost dimension. Since there is no direct comparability in evaluating a specific cost individually, this paper takes the sum of the associated costs to manifest the overall cost competitiveness of representative car-sharing operators in each city.

In the second step of weighting, the assigned evaluation points for secondary indexes will be weighted within the primary indexes by the entropy weight method (EWM) and then be weighted to obtain the primary index score. EWM is an objective weighting method. Its basic idea is to determine the weight of the index based on the information entropy contained in the index sample [17]. Generally speaking, if an index has a larger information entropy, it means that this index can provide more information and can play a greater role in comprehensive evaluation and pattern recognition. That's why the index is assigned with greater weight [18]. The specific calculation method is shown as follows.

(1) Normalization processing

After normalization processing of standardized index evaluation points to calculate the proportion of the corresponding secondary index evaluation point $\left(X_{i j}^{(k)}\right)$ among the point for all the seven cities with the $j^{\text {th }}$ secondary index of the $\mathrm{i}^{\text {th }}$ primary index and the $\mathrm{k}^{\text {th }}$ city:

$$
P_{i j}^{(k)}=\frac{X_{i j}^{(k)}}{\sum_{k=1}^{7} X_{i j}^{(k)}}
$$

(2) Weight distribution

The $P_{i j}^{(k)}$ above is the normalized evaluation point for the secondary index, which corresponds to the information entropy as follows:

$$
E_{i j}=-q \sum_{k=1}^{7} P_{i j}^{(k)} \ln P_{i j}^{(k)}, q=\frac{1}{\ln 7}
$$

It should be noted that when $P_{i j}^{(k)}=0$, then $P_{i j}^{(k)} \ln P_{i j}^{(k)}=0$. Furthermore, $\mathrm{q}$ is the adjustment coefficient, related to the urban sample size (herein $\mathrm{q}=$ $0.514)$.

Translate the information entropy into the weight corresponding to the evaluation point for the $\mathrm{j}^{\text {th }}$ secondary index:

$$
w_{i j}=\frac{1-E_{i j}}{f(i)-\sum_{j=1}^{f(i)} E_{i j}}
$$

In the formula, $f(i)$ represents the number of evaluation points for the secondary index included in the $\mathrm{i}^{\text {th }}$ primary index, and $1 \leq j \leq f(i)$.

(3) Calculation of primary indexes 
$Y_{i}^{(k)}$, which means the $\mathrm{i}^{\text {th }}$ primary index in the $\mathrm{k}^{\text {th }}$ city, is the sum of the products of normalized evaluation points for the secondary index $\left(X_{i 1}^{(k)}, X_{i 2}^{(k)}, \ldots, X_{i j}^{(k)}\right)$ with the corresponding weights $\left(w_{i 1}, w_{i 2}, \ldots, w_{i j}\right)$ :

$$
Y_{i}^{(k)}=\sum_{j=1}^{f(i)} X_{i j}^{(k)} w_{i j}
$$

As for the primary indexes of the cost dimension, they can be directly ranked by calculated values reflecting overall cost competitiveness.

\section{Result and discussion}

\subsection{Independent evaluation of strengths and weaknesses of car-sharing development by cities}

According to the evaluation standards and calculation methods of various second-level index points in all dimensions, the work concluding data collection, information classification, research and analysis, measurement, and evaluation around the seven chosen cities gets finished. First, the evaluation points of each second-level index are formed, and then the evaluation indexes are weighted hierarchically according to the weighting principle, and finally the evaluation results of the first-level indexes of seven cities can be obtained. In order to form a comprehensive understanding of the car sharing development in each city, this paper determines the weight of the first-level indicators according to the importance of each first-level indicator to obtain the overall score of each city by weighting. The original intention of this paper is to select sample cities for the development of a national car-sharing development level evaluation based on independent research, so the firstlevel indicators and overall scores of all cities are arithmetically averaged according to the number of cities, so as to obtain the first-level overall score of various firstlevel indicator across the country.

Radar charts can more clearly show the advantages and disadvantages of various cities. According to the absolute value and balance of all indicators, the seven cities can be divided into four categories: the first category includes Shanghai, the second includes Shenzhen and Guangzhou, the third includes Hangzhou and Wuhu, and the fourth includes Beijing and Chongqing.

As shown in Figure 1, the development of car sharing in Shanghai is relatively balanced and all index values are high except the cost competitiveness. To be specific, the effectiveness of business models, policy support and technological advancement are highlighted, which reflects great business atmosphere, meticulous management of government departments, and the strength of automobile enterprises in Shanghai. The high cost of Shanghai car sharing stems from its high-standard service positioning, which will be more profitable after the concept of car sharing is deeply rooted in people's hearts, but it might not be very suitable for the current stage of car sharing.
As shown in Figure 2 and Figure 3, the car-sharing development is also relatively balanced in Shenzhen and Guangzhou, but most of the first-level indicators are lower than those in Shanghai. In addition, these two cities share similar advantages and disadvantages. Specifically, the two cities both have advantages in cost competitiveness and technological advancement, while have disadvantages in products and service experience. Since Shenzhen and Guangzhou are geographically near, so the same characteristics of local policies, urban management, and regional culture have a similar impact on the development of local car sharing. On the basis of fully understanding the value of car sharing, the managers of the two cities can learn from the policy formulation of Shanghai city managers and launch more targeted support policies.

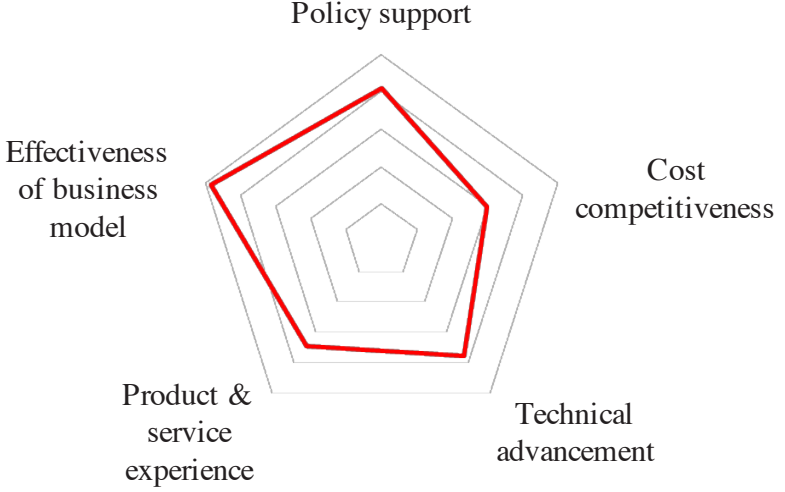

Figure 1. Evaluation of the car-sharing development in Shanghai

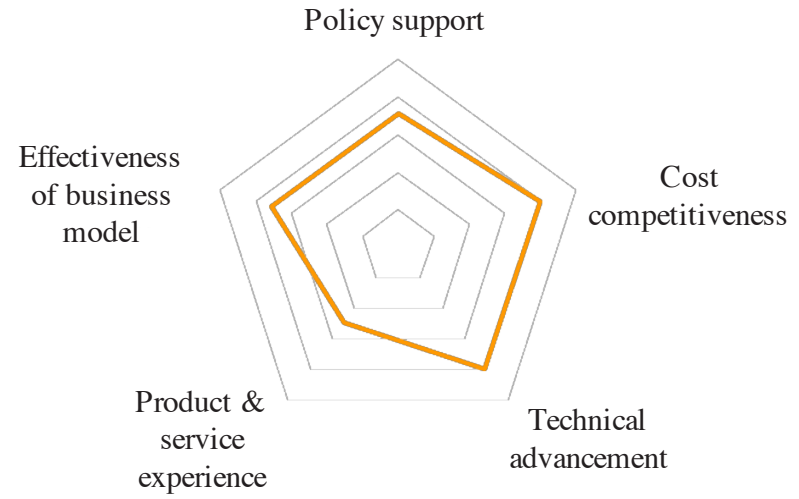

Figure 2. Evaluation of the car-sharing development in Guangzhou

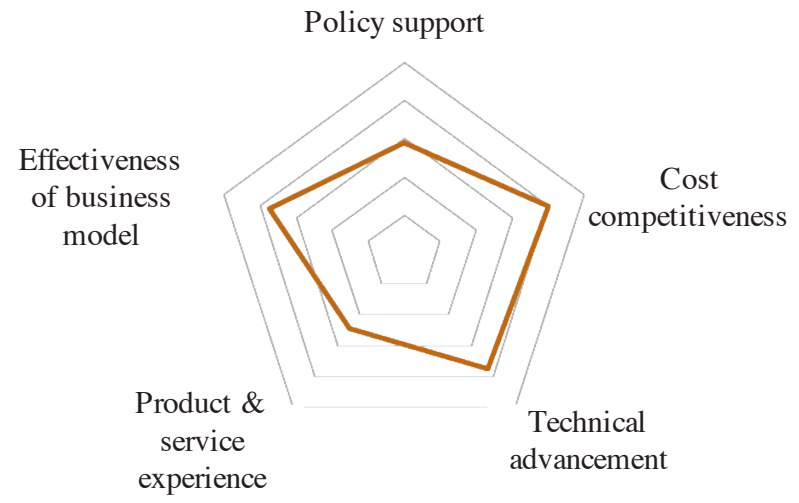

Figure 3. Evaluation of the car-sharing development in Shenzhen 
As shown in Figure 4 and Figure 5, although the balance of car-sharing development in Hangzhou and Wuhu is great, the scores of most first-level indicators are smaller than those of Shenzhen and Guangzhou, which indicates slightly inferior overall development level. Although Hangzhou and Wuhu are geographically close as well, they do not show the same convergence as Shenzhen and Guangzhou since the two cities are both located in the Yangtze River Delta region. That is why Hangzhou has advantages in business model effectiveness (which is related to the local dynamic economic structure) and Wuhu has better performance in policy support, products and service experience (which reflects the local government's strong support for automobile-related industries). The relatively small urban area and population scale of Wuhu can facilitate the development of high-quality services. Similarly, Hangzhou's management policy should also be more developed and effective. Considering the continuous development of Hangzhou in the future, car sharing will have a greater role in urban transportation. Wuhu has already demonstrated strong enough policy support, but the technological research and development capabilities of local car companies must be more up-to-date, in order to adapt to the continuous upgrade of car sharing in the future.

As shown in Figure 6 and Figure 7, Beijing and Chongqing deliver moderate balance of local car-sharing development. Belonging to the same category does not mean sharing similar development levels for these two cities. In terms of cost competitiveness, technical advancement and effectiveness of business models, Beijing occupies a leading position among all the seven cities but lags far behind in products and services. Such factors as a large number of emerging enterprises, developed local internet industry, high sensitivity to the promising industries, and excessive competition in Beijing may adversely affect the lean operation of the industry. Except in effectiveness of business models and technical advancement, Chongqing does not have advantages in other aspects. As the only western city in this paper, Chongqing faces certain differences with the eastern areas in terms of management philosophy of city managers and operation level of enterprises, albeit its positioning as an economic center in Southwest China.

\section{Policy support}

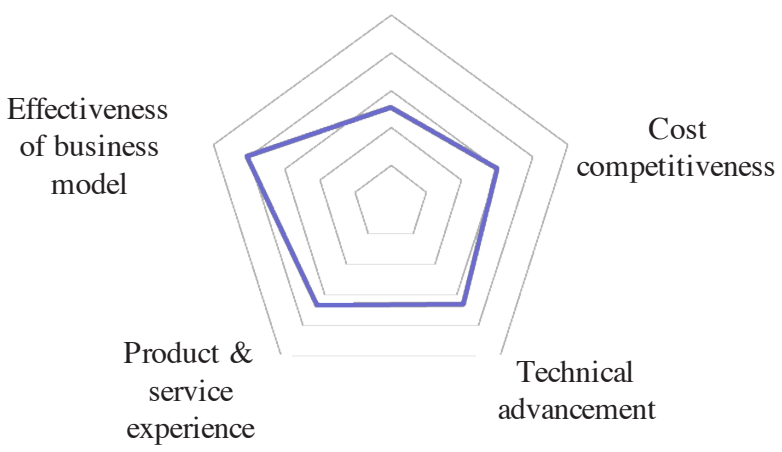

Figure 4. Evaluation of the car-sharing development in Hangzhou

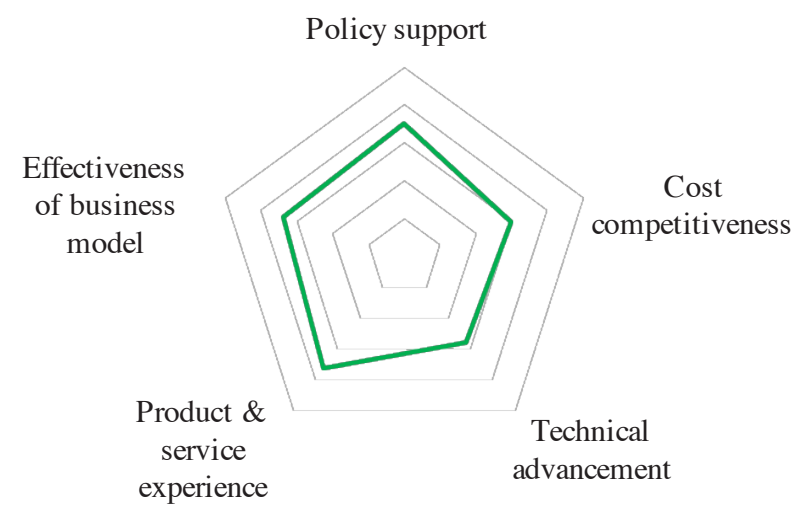

Figure 5. Evaluation of the car-sharing development in Wuhu Policy support

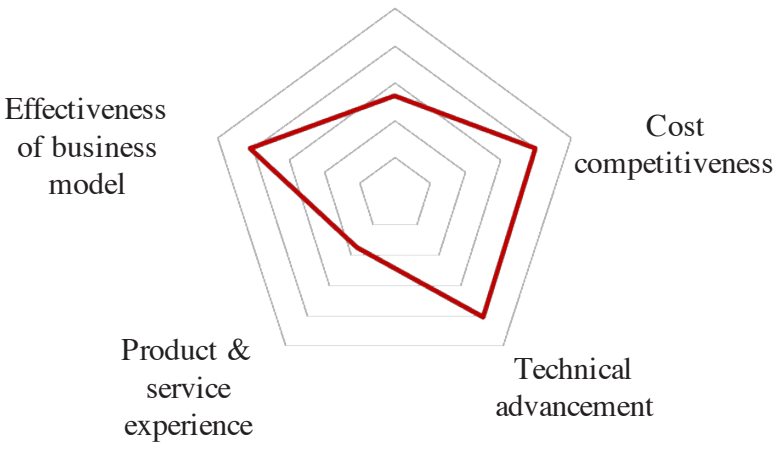

Figure 6. Evaluation of the car-sharing development in Beijing Policy support

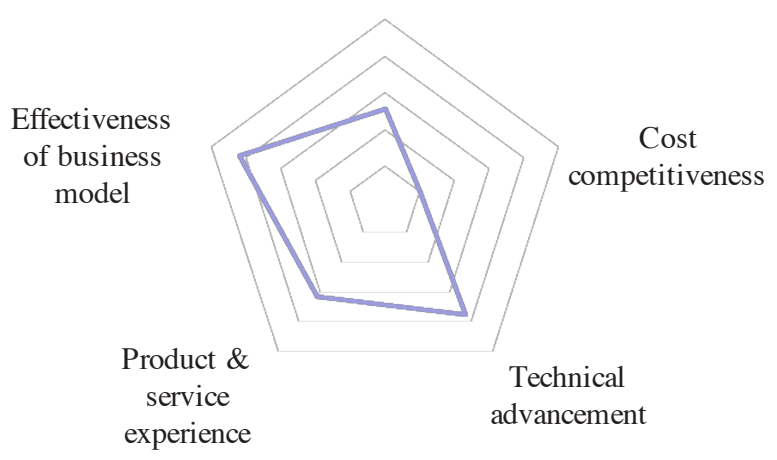

Figure 7. Evaluation of the car-sharing development in Chongqing

Car-sharing development varies by cities, which indicates that the development of the car sharing industry is still under exploration. There are no sophisticated and adequate fixed paradigms for whether policy formulation, cost control and technology application, or service and business cooperation. Although there are geographical, economic, and other objective fundamental differences among regions that cannot be eliminated in a short term, on the one hand, each city needs to fill up shortcomings quickly, on the other hand, a nationwide successful business model should have some characteristics and strategies that can be replicated regardless of geographical locations. Such a chaotic pattern does bring strong car-sharing service operators opportunities for trial and exploration, but this paper believes that calm observance, abstraction of common points, follow the law of development, and forward-looking prejudgment will make the car sharing industry develop faster and better. 


\subsection{Comparion of car sharing development from national level and comprehensive evaluation}

The total scores of all the seven cities serve as good demonstrations. Because the total score is obtained by weighting the primary indexes according to specific criteria, which is based on the importance of such indexes for industry development. The ranking based on the total score of each city is shown in Figure 8. It is easy to observe that the ranking pattern from the perspective of total score has a certain similarity to the classification results from the perspective of the balance of the aforementioned dimensions. That is, Shanghai ranks the first, Guangzhou, Wuhu, Shenzhen, and Hangzhou are in one segment, and Beijing and Chongqing are in another. It is not hard to explain such a "coincidence", because there is no absolute difference in the development level between these cities. That is why the balance of development is more important.

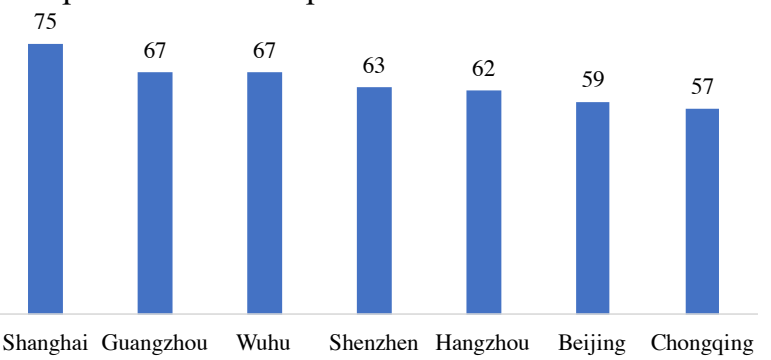

Figure 8. Ranking of car-sharing development by cities

The scores of the primary indexes initially reflect the overall level of car sharing development in China. It can be found from Figure 9 that the total score of 64 points means "being barely passed", and there is huge space for improvement. Good development of business model effectiveness reflects that innovation of business models led by car sharing indeed drew great attention from related industries and enterprises over a period of time. In addition, the expansion of business cooperation must be supported by stable and continuous basic car sharing services. Therefore, specific suggestions to the effectiveness of business models will not be further discussed in this paper.

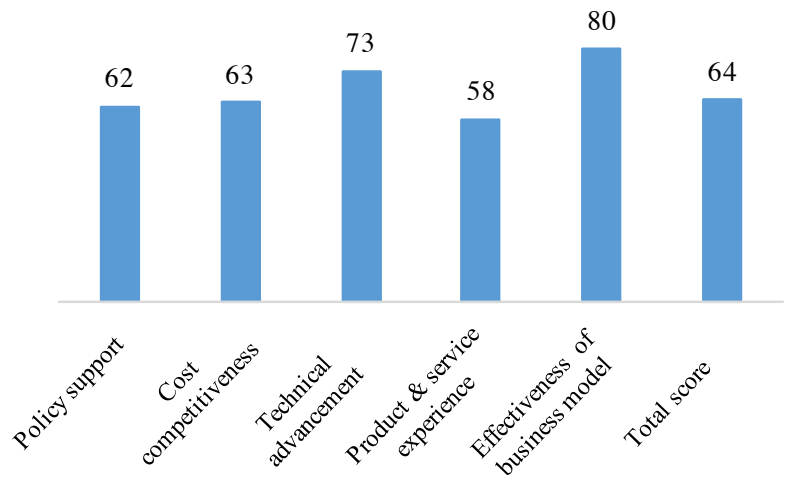

Figure 9. Evaluation of car-sharing development in China

Although technical advancement occupied the second place of the ranking, it does not mean that technology application in the car sharing industry has already been hassle-free. Automobile enterprises are now dedicated to the $R \& D$ of autonomous driving and new energy technologies, with the potential for future progress. The second place is obtained from the comparison with the other three development shortcomings. In fact, the industry development is still hindered by many technological factors. Product and service experience is the biggest weakness, which will bring hidden dangers to the sound development of the industry. Weak policy support and cost competitiveness also reflect the cost-topolicy sensitivity of the car sharing industry. Initial suggestions on how to make improvement in the four aspects will be provided below.

\section{Conclusion}

In the process of specifically determining the research object of the car sharing industry, it is found that the development of car sharing is affected by urban areas and corporate strategies. Car sharing is an asset-heavy business model, which requires the precondition of owning vehicles and parking spaces. Therefore, local governments' policy support for the purchase of NEVs, the construction of outlets, and infrastructure can affect the operating costs. The capacity and specific decisions of enterprises in terms of vehicle scale, vehicle scheduling, and business cooperation will affect the level of car sharing service. There are a group of representative operators with large scale, wide coverage, and solid user base that have survived the competition in the car sharing market.

Use the car-sharing development evaluation index system to systematically evaluate the selected representative cities. Local car-sharing industries in the representative city have different strengths and weaknesses, but no one has occupied an absolutely leading position. Whether in terms of the balance of development or the scores of primary indexes, Shanghai, represented by EVCARD, has a relatively developed carsharing industry.

On the whole, there are great differences between the car-sharing development level in these cities. Firstly, there is no consistent ranking of the scores of the five primary indexes for the seven cities. In other words, there is no absolute level of maturity of the five primary indexes. For instance, the score of policy support is higher than that of product and service experience in Beijing, while the situation is opposite in Hangzhou. On the one hand, it shows that development strategies vary by cities and enterprises. On the other hand, the five primary indexes are relatively independent, without obvious correlation.

Secondly, the balance degrees of car sharing development are also different in the seven cities. According to the results, Shanghai seems to be the most balanced development of car sharing, followed by Hangzhou, Wuhu, Guangzhou, and Shenzhen, while Beijing and Chongqing have obvious shortcomings. Regardless of any technology, any enterprise, or any industry, balance is an important dimension for the judgment of development results. Because the stronger the balance is, the greater the joint force will be. On the contrary, obvious shortcomings will become constraints, which is the core of the "buckets effect". 
Finally, the highest value of every primary index does not centralize in one or two cities, which means no city has absolute leading positions in all dimensions. For example, Shanghai has the highest score of policy support, while Beijing has the highest score of cost competitiveness, and Wuhu has the highest score of product and service experience. It means that the carsharing development of all these cities are worth learning from.

Generally speaking, the product and service experience is the weakest link in the car-sharing industry. The lowest score of product and service experience reflects that existing operators do not pay enough attention to such an index. As a service model, users' acceptance and experience should be the focus. The shortcomings should be improved in the future. Meanwhile, policy support and cost competitiveness performance are not optimistic, which indicates that the car sharing based on asset-heavy model has limited capabilities in cost control and profit increase without incentive policies.

\section{Suggestion}

The car-sharing industry's development requires cooperation between governments and enterprises, as well as technologies for driving force. Government resources and support are crucial to the car sharing based on an asset-heavy business model, in which more efforts should be made. Land use, license plates, vehicle purchase, and infrastructure that affect the supply capacity of the car sharing industry can all become major policy concerns. Of course, the government also needs to carry out strict supervision and dynamical adjustment of the management rules for the car sharing industry which is in the period of exploration.

Technology will become the core of improving or even disrupting service level, so more efforts should be made in the R\&D to realize business applications as soon as possible. As a component of the connectivity, automation, sharing and electrification (C.A.S.E.) in the automotive industry, car sharing requires coordination and mutual promotion with automation, connectivity, and electrification for higher level. Only if more breakthroughs are made in the other three aspects earlier, sharing will face fewer objective constraints for penetration and users' demand for car sharing experience will also be better satisfied.

Enterprises should establish the concept of "community of shared benefits" with users and strengthen the review and improvement of their business from the perspective of users. Cost control is one of the business goals, but enterprises should have long-term strategic thinking instead of just focusing on short-term cost reduction. It requires enterprises to truly improve service thinking and awareness and enhance service quality and level to gain long-term trust and support from users, forming a long-term car sharing ecosystem moving towards a good future.

\section{Acknowledgement}

(This research is funded by the following two projects:

1. The key consulting research project of the Chinese Academy of Engineering "The core bottleneck of smart cars and intelligent traffic management": 2019-XZ-55, 2019.12.16-2020.12.15

2. The key consulting research project of the Chinese Academy of Engineering "The deep integration development strategy of China's smart city, smart transportation and smart car": 2019-XZ-4, 2019-03-082021-03-08)

\section{References}

1. CASS, The 17th Report of Chinese Urban Competitiveness (2019) (in Chinese)

http://www.cssn.cn/zx/201906/t20190624_4922401.s html

2. M. Chen, F. Zhao, Z Liu, Proceedings of SAE-China Congress 2018, 2170-2174 (2018)

3. J. Lan, D. Chu, China Population, Resources and Environment, 11, 98-105 (2016) (in Chinese)

4. M. Zhao, S. Wang, Journal of Dalian University of Technology (Social Sciences), 39, 3, 32-38 (2018) (in Chinese)

5. Panda Capital, Analysis of Car Sharing Market and Financial Model (2017) (in Chinese) https://36kr.com/coop/zaker/5062446.html

6. M. Namazu, H. Dowlatabadi, Transp. Policy, 64, 3850 (2018)

7. S. Le Vine, J. Polak, Transp. Policy, 75, 119-127 (2004)

8. J. Chen, F. Cao, Journal of Xichang University (Natural Science Edition), 3, 84-87 (2018) (in Chinese)

9. X. Dai, Science and Information, 19, 144-145 (2018) (in Chinese)

10. J. Firnkorn, M. Müller, ECOL.ECON. 70, 1519-1528 (2011)

11. E. W. Martin, S. A. Shaheen, J. Lidicker, Carsharing's Impact on Household Vehicle Holdings: Results from a North American Shared-Use Vehicle Survey (2010)

12. F. Zhao, F. Liu, Z. Liu, H. Hao, Strategic Study of CAE, 20, 1, 104-112 (2018) (in Chinese)

13. H. Becker, A. Loder, B. Schmid, K. W. Axhausen, Travel Behav. Soc. 8, 26-36 (2017)

14. F. Zhao, Z. Liu, K. Yang, Q Ma, Automotive Technology Innovation (2019) (in Chinese)

15. X. Wang, J. Wang, Auto Time, 14, 8-9 (2019) (in Chinese)

16. Z. Liu, M. Chen, F. Zhao, Enterprise Economy, 7, 44-48 (2015) (in Chinese)

17. X. Zhu, Fundamentals of Applied Information Theory (2001) (in Chinese)

18. S. Zhang, M. Zhang, G. Chi, Chinese Journal of Management, 7, 1, 34-42 (2010) (in Chinese) 